EPJ Web of Conferences 73, 03006 (2014)

DOI: $10.1051 /$ epjconf/20147303006

(C) Owned by the authors, published by EDP Sciences, 2014

\title{
Chiral-scale perturbation theory about an infrared fixed point
}

\author{
R.J. Crewther ${ }^{1, \mathrm{a}}$ and Lewis C. Tunstall ${ }^{1,2, \mathrm{~b}, \mathrm{c}}$ \\ ${ }^{1}$ CSSM and ARC Centre of Excellence for Particle Physics at the Tera-scale, Department of Physics, \\ University of Adelaide, Adelaide SA 5005, Australia \\ ${ }^{2}$ Albert Einstein Centre for Fundamental Physics, Institute for Theoretical Physics, University of Bern, \\ Sidlerstrasse 5, 3012 Bern, Switzerland
}

\begin{abstract}
We review the failure of lowest order chiral $S U(3)_{L} \times S U(3)_{R}$ perturbation theory $\chi \mathrm{PT}_{3}$ to account for amplitudes involving the $f_{0}(500)$ resonance and $O\left(m_{K}\right)$ extrapolations in momenta. We summarize our proposal to replace $\chi \mathrm{PT}_{3}$ with a new effective theory $\chi \mathrm{PT}_{\sigma}$ based on a low-energy expansion about an infrared fixed point in 3 -flavour QCD. At the fixed point, the quark condensate $\langle\bar{q} q\rangle_{\text {vac }} \neq 0$ induces nine NambuGoldstone bosons: $\pi, K, \eta$ and a QCD dilaton $\sigma$ which we identify with the $f_{0}(500)$ resonance. We discuss the construction of the $\chi \mathrm{PT}_{\sigma}$ Lagrangian and its implications for meson phenomenology at low-energies. Our main results include a simple explanation for the $\Delta I=1 / 2$ rule in $K$-decays and an estimate for the Drell-Yan ratio in the infrared limit.
\end{abstract}

\section{Three-flavor chiral expansions: Problems in the scalar-isoscalar channel}

Chiral $S U(3)_{L} \times S U(3)_{R}$ perturbation theory $\chi \mathrm{PT}_{3}$ is nowadays well established as the framework to systematically analyze the low-energy interactions of $\pi, K, \eta$ mesons — the pseudo Nambu-Goldstone (NG) bosons of approximate chiral symmetry. The method relies on expansions about a NG-symmetry, viz., low-energy scattering amplitudes and matrix elements can be described by an asymptotic series

$$
\mathcal{A}=\left\{\mathcal{A}_{\mathrm{LO}}+\mathcal{A}_{\mathrm{NLO}}+\mathcal{A}_{\mathrm{NNLO}}+\cdots\right\}_{\chi \mathrm{PT}}
$$

in powers and logarithms of $O\left(m_{K}\right)$ momentum and quark masses $m_{u, d, s}=O\left(m_{K}^{2}\right)$, with $m_{u, d} / m_{s}$ held fixed. The scheme works provided that contributions from the NG sector $\{\pi, K, \eta\}$ dominate those from the non-NG sector $\{\rho, \omega, \ldots\}$; an assumption known as the partial conservation of axial current (PCAC) hypothesis.

It has been observed [1], however, that the $\chi \mathrm{PT}_{3}$ expansion (1) is afflicted with a peculiar malady: it typically diverges for amplitudes which involve both a $0^{++}$channel and $O\left(m_{K}\right)$ extrapolations in

\footnotetext{
ae-mail: rcrewthe@physics.adelaide.edu.au

be-mail: tunstall@itp.unibe.ch

${ }^{\mathrm{c}}$ Speaker.
}

This is an Open Access article distributed under the terms of the Creative Commons Attribution License 4.0, which permits unrestricted use, distribution, and reproduction in any medium, provided the original work is properly cited. 

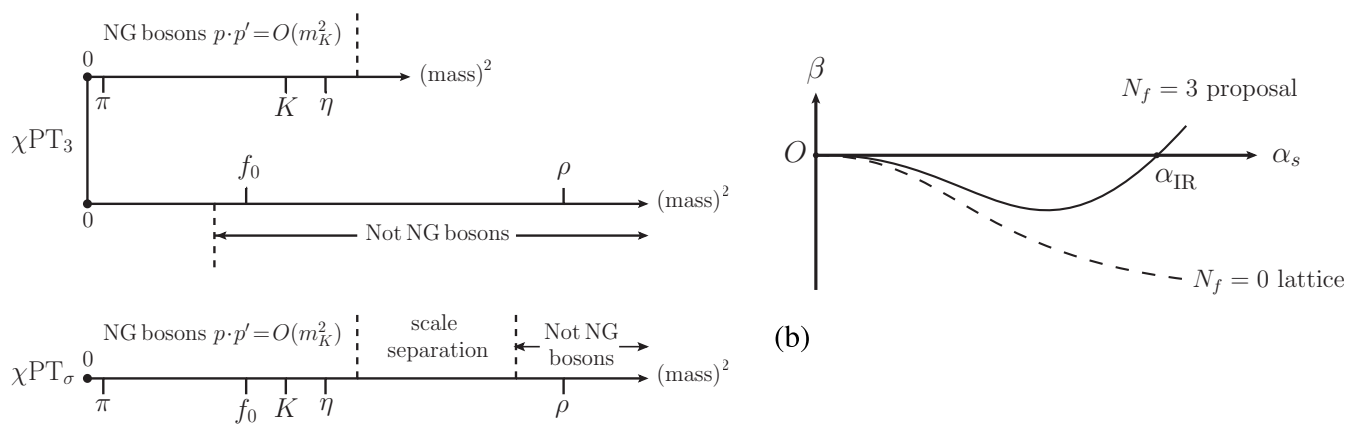

(b)

(a)

Figure 1. (a) Scale separations between Nambu-Goldstone (NG) sectors and other hadrons for each type of chiral perturbation theory $\chi \mathrm{PT}$ discussed in this proceeding. In conventional three-flavor theory $\chi \mathrm{PT}_{3}$ (top diagram), there is no scale separation: the non-NG boson $f_{0}(500)$ sits in the middle of the NG sector $\{\pi, K, \eta\}$. Our three-flavor proposal $\chi \mathrm{PT}_{\sigma}$ (bottom diagram) for $O\left(m_{K}\right)$ extrapolations in momenta implies a clear scale separation between the NG sector $\left\{\pi, K, \eta, \sigma=f_{0}\right\}$ and the non-NG sector $\left\{\rho, \omega, K^{*}, N, \eta^{\prime}, \ldots\right\}$. (b) Proposed $\beta$-function (solid line) for $N_{f}=3$ flavor QCD with infrared fixed point $\alpha_{\mathrm{IR}}$. The dashed line shows the Yang-Mills $\left(N_{f}=0\right)$ lattice result [6] for continued growth in $\alpha_{s}$ with decreasing scale $\mu$. Despite extensive literature [7] concerning the existence of $\alpha_{\mathrm{IR}}$, there is currently no consensus which of the above two, physically distinct, scenarios is actually realized in QCD. In particular, it is unclear how sensitive existing results are to variations in $N_{f}$. This is perhaps unsurprising, since modern calculations utilize different, nonperturbative definitions of $\alpha_{s}$, thereby making comparisons between various analyses difficult.

momenta. The origin of this phenomenon can be traced to the $f_{0}(500)$ resonance, a broad $0^{++}$state whose complex pole mass and residue [2]

$$
m_{f_{0}}=441-i 272 \mathrm{MeV} \text { and }\left|g_{f_{0} \pi \pi}\right|=3.31 \mathrm{GeV}
$$

have been determined to remarkable precision. Since $\chi \mathrm{PT}_{3}$ classes $f_{0}$ pole terms as next-to-leading order (NLO), figure 1a shows why the low-energy expansion (1) fails: the location of $f_{0}$ and its strong coupling to $\pi, K, \eta$ mesons invalidates the requirements of PCAC.

\section{Three-flavor chiral-scale expansions about an infrared fixed point}

In this proceeding, we summarize our proposal [3] to solve the convergence problem of $\chi \mathrm{PT}_{3}$ expansions (1) by modifying the leading order (LO) of the 3-flavor theory. In short, our solution involves extending the standard NG sector $\{\pi, K, \eta\}$ to include $f_{0}(500)$ as a QCD dilaton $\sigma$ associated with the spontaneous breaking of scale invariance. The scale symmetric counterpart of PCAC - partial conservation of dilatation current (PCDC) - then implies that amplitudes with $\sigma / f_{0}$ pole terms dominate, compared with contributions from the non-NG sector $\left\{\rho, \omega, K^{*}, N, \eta^{\prime}, \ldots\right\}{ }^{1}$

This scenario can occur in QCD if at low energy scales $\mu \ll m_{t, b, c}$, the strong coupling $\alpha_{s}$ for the 3 -flavor theory runs nonperturbatively to an infrared fixed point $\alpha_{\mathrm{IR}}$ (Fig. 1b). At the fixed point, the gluonic term in the strong trace anomaly [9]

$$
\theta_{\mu}^{\mu}=\frac{\beta\left(\alpha_{s}\right)}{4 \alpha_{s}} G_{\mu \nu}^{a} G^{a \mu \nu}+\left(1+\gamma_{m}\left(\alpha_{s}\right)\right) \sum_{q=u, d, s} m_{q} \bar{q} q
$$

\footnotetext{
${ }^{1}$ A discussion on violations of PCDC and Weinberg's power counting scheme [8] in $\gamma \gamma$ channels is contained in [3].
} 
vanishes, which implies that in the chiral limit

$$
\left.\theta_{\mu}^{\mu}\right|_{\alpha_{s}=\alpha_{\mathrm{IR}}}=\left(1+\gamma_{m}\left(\alpha_{\mathrm{IR}}\right)\right)\left(m_{u} \bar{u} u+m_{d} \bar{d} d+m_{s} \bar{s} s\right) \rightarrow 0,
$$

and thus $\langle\bar{q} q\rangle_{\text {vac }}$ acts as a condensate for both scale and chiral $S U(3)_{L} \times S U(3)_{R}$ transformations. ${ }^{2}$ By considering infrared expansions about the combined limit

$$
m_{u, d, s} \sim 0 \quad \text { and } \quad \alpha_{s} \lesssim \alpha_{\mathrm{IR}},
$$

our proposal is to replace $\chi \mathrm{PT}_{3}$ by chiral-scale perturbation theory $\chi \mathrm{PT}_{\sigma}$, where the strange quark mass $m_{s}$ in (4) sets the scale of $m_{f_{0}}^{2}$ as well as $m_{K}^{2}$ and $m_{\eta}^{2}$ (figure 1a, bottom diagram). As a result, the rules for counting powers of $m_{K}$ are changed: $f_{0}$ pole amplitudes (NLO in $\chi \mathrm{PT}_{3}$ ) are promoted to LO. That fixes the LO problem for amplitudes involving $0^{++}$channels and $O\left(m_{K}\right)$ extrapolations in momenta. Note that we achieve this without upsetting successful LO $\chi \mathrm{PT}_{3}$ predictions for amplitudes which do not involve the $f_{0}$; that is because the $\chi \mathrm{PT}_{3}$ Lagrangian equals the $\sigma \rightarrow 0$ limit of the $\chi \mathrm{PT}_{\sigma}$ Lagrangian.

In the physical region $0<\alpha_{s}<\alpha_{\mathrm{IR}}$, the effective theory consists of operators constructed from the $S U(3)$ field $U=U(\pi, K, \eta)$ and chiral invariant dilaton $\sigma$, with terms classified by their scaling dimension $d$ :

$$
\mathcal{L}_{\chi \mathrm{PT}_{\sigma}}=\mathcal{L}\left[\sigma, U, U^{\dagger}\right]=: \mathcal{L}_{\text {inv }}^{d=4}+\mathcal{L}_{\text {anom }}^{d>4}+\mathcal{L}_{\text {mass }}^{d<4}: .
$$

Explicit formulas for the strong, weak, and electromagnetic interactions are obtained by scaling Lagrangian operators such as $\mathcal{K}\left[U, U^{\dagger}\right]=\frac{1}{4} F_{\pi}^{2} \operatorname{Tr}\left(\partial_{\mu} U \partial^{\mu} U^{\dagger}\right)$ and $\mathcal{K}_{\sigma}=\frac{1}{2} \partial_{\mu} \sigma \partial^{\mu} \sigma$ by appropriate powers of the $d=1$ field $e^{\sigma / F_{\sigma}}$. For example, the LO strong Lagrangian reads

$$
\begin{aligned}
\mathcal{L}_{\text {inv, LO }}^{d=4} & =\left\{c_{1} \mathcal{K}+c_{2} \mathcal{K}_{\sigma}+c_{3} e^{2 \sigma / F_{\sigma}}\right\} e^{2 \sigma / F_{\sigma}}, \\
\mathcal{L}_{\text {anom }, \mathrm{LO}}^{d>4} & =\left\{\left(1-c_{1}\right) \mathcal{K}+\left(1-c_{2}\right) \mathcal{K}_{\sigma}+c_{4} e^{2 \sigma / F_{\sigma}}\right\} e^{\left(2+\beta^{\prime}\right) \sigma / F_{\sigma}}, \\
\mathcal{L}_{\text {mass, } \mathrm{LO}}^{d<4} & =\operatorname{Tr}\left(M U^{\dagger}+U M^{\dagger}\right) e^{\left(3-\gamma_{m}\right) \sigma / F_{\sigma}},
\end{aligned}
$$

where $F_{\sigma} \approx 100 \mathrm{MeV}$ is the dilaton decay constant, whose value is estimated by applying an analogue of the Goldberger-Treiman relation to analyses of $N N$-scattering [10]. Here the anomalous dimensions $\gamma_{m}=\gamma_{m}\left(\alpha_{\mathrm{IR}}\right)$ and $\beta^{\prime}=\beta\left(\alpha_{\mathrm{IR}}\right)$ are evaluated at the fixed point because we expand in $\alpha_{s}$ about $\alpha_{\mathrm{IR}}$. The low-energy constants $c_{1}$ and $c_{2}$ are not fixed by symmetry arguments alone, while vacuum stability in the $\sigma$ direction implies that both $c_{3}$ and $c_{4}$ are $O(M)$. From (7), one obtains formulas for the dilaton mass $m_{\sigma}$

$$
m_{\sigma}^{2} F_{\sigma}^{2}=F_{\pi}^{2}\left(m_{K}^{2}+\frac{1}{2} m_{\pi}^{2}\right)\left(3-\gamma_{m}\right)\left(1+\gamma_{m}\right),-\beta^{\prime}\left(4+\beta^{\prime}\right) c_{4}
$$

and $\sigma \pi \pi$ coupling

$$
\mathcal{L}_{\sigma \pi \pi}=\left\{\left(2+\left(1-c_{1}\right) \beta^{\prime}\right)|\partial \pi|^{2}-\left(3-\gamma_{m}\right) m_{\pi}^{2}|\pi|^{2}\right\} \sigma /\left(2 F_{\sigma}\right) .
$$

Note that (9) is derivative, so an on-shell dilaton is $O\left(m_{\sigma}^{2}\right)$ and consistent with $\sigma$ being the broad resonance $f_{0}(500)$.

Our proposed replacement for $\chi \mathrm{PT}_{3}$ possesses some desirable features, the foremost being:

1. The $\Delta I=1 / 2$ rule for $K$-decays emerges as a consequence of $\chi \mathrm{PT}_{\sigma}$, with a dilaton pole diagram (figure 2a) accounting for the large $I=0$ amplitude in $K_{S} \rightarrow \pi \pi$. Here, vacuum alignment [13] of the effective potential induces an interaction $\mathcal{L}_{K_{S} \sigma}=g_{K_{S} \sigma} K_{S} \sigma$ which mixes $K_{S}$ and $\sigma$ in LO. The effective coupling $g_{K_{S} \sigma}$ is fixed by data on $\gamma \gamma \rightarrow \pi^{0} \pi^{0}$ and $K_{S} \rightarrow \gamma \gamma$, with our estimate $\left|g_{K_{S} \sigma}\right| \approx 4.4 \times 10^{3} \mathrm{keV}^{2}$ accurate to a precision $\lesssim 30 \%$ expected from a 3-flavor expansion.

\footnotetext{
2 The former property is a simple consequence of the fact the $\bar{q} q$ is not a singlet under dilatations. The dual role of $\langle\bar{q} q\rangle_{\text {vac }}$ was explored $[4,5]$ in some detail prior to the advent of QCD.
} 


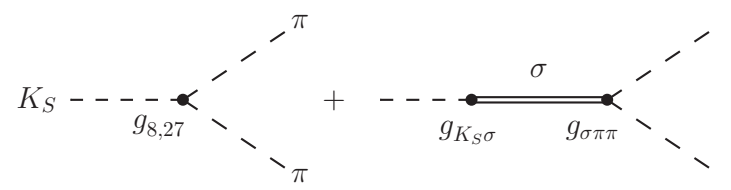

(a)

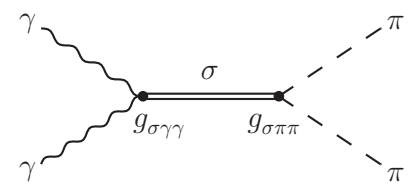

(b)

Figure 2. (a) Tree diagrams in the effective theory $\chi \mathrm{PT}_{\sigma}$ for the decay $K_{S} \rightarrow \pi \pi$. The vertex amplitudes due to 8 and 27 contact couplings $g_{8}$ and $g_{27}$ are dominated by the $\sigma / f_{0}$-pole amplitude. The magnitude of $g_{K_{S} \sigma}$ is found by applying $\chi \mathrm{PT}_{\sigma}$ to $K_{S} \rightarrow \gamma \gamma$ and $\gamma \gamma \rightarrow \pi \pi$. (b) Dilaton pole in $\gamma \gamma \rightarrow \pi \pi$. Lowest order $\chi \mathrm{PT}_{\sigma}$ includes other tree diagrams (for $\pi^{+} \pi^{-}$production) and also $\pi^{ \pm}, K^{ \pm}$loop diagrams (suppressed by a factor $1 / N_{c}$ ) coupled to both photons.

Combined with data for the $f_{0}$ width (Eq. (2)), we find an amplitude $\left|A_{\sigma-\text { pole }}\right| \approx 0.34 \mathrm{keV}$ which accounts for the large magnitude $\left|A_{0}\right|_{\text {expt. }}=0.33 \mathrm{keV}$. Consequently, the $\mathrm{LO}$ of $\chi \mathrm{PT}_{\sigma}$ explains the $\Delta I=1 / 2$ rule for kaon decays.

2. Our analysis of $\gamma \gamma$ channels and the electromagnetic trace anomaly [11, 12] yields a relation between the effective $\sigma \gamma \gamma$ coupling and the nonperturbative Drell-Yan ratio $R_{\mathrm{IR}}$ at $\alpha_{\mathrm{IR}}$ :

$$
g_{\sigma \gamma \gamma}=\frac{2 \alpha}{3 \pi F_{\sigma}}\left(R_{\mathrm{IR}}-\frac{1}{2}\right) .
$$

A phenomenological value for $R_{\mathrm{IR}}$ is deduced by considering $\gamma \gamma \rightarrow \pi^{0} \pi^{0}$ in the large- $N_{c}$ limit (Fig. 2b). Dispersive analyses [14] of this processes are able to determine the radiative width of $f_{0}(500)$, which in turn constrains $g_{\sigma \gamma \gamma}$ and yields the estimate $R_{\mathrm{IR}} \approx 5$.

\section{References}

[1] U.-G. Meissner, Comments Nucl. Part. Phys. 20, 119 (1991); Rep. Prog. Phys. 56, 903 (1993)

[2] I. Caprini, G. Colangelo, and H. Leutwyler, Phys. Rev. Lett. 96, 132001 (2006)

[3] R.J. Crewther and L.C. Tunstall, arXiv:1312.3319 [hep-ph]; arXiv:1203.1321 [hep-ph]

[4] J. Ellis, Nucl. Phys. B 22, 478 (1970)

[5] R.J. Crewther, Phys. Lett. B 33, 305 (1970)

[6] M. Lüscher, R. Sommer, P. Weisz, and U. Wolff, Nucl. Phys. B 413, 481 (1994)

[7] T. Banks and A. Zaks, Nucl. Phys. B 196, 189 (1982); L. von Smekal, A. Hauck and R. Alkofer, Phys. Rev. Lett. 79, 3591 (1997); C.S. Fischer and R. Alkofer, Phys. Rev. D 67, 094020 (2003); A.C. Aguilar, D. Binosi and J. Papavassiliou, J. High Energy Phys. 07, 002 (2010); S.J. Brodsky, G.F. de Teramond and A. Deur, Phys. Rev. D 81, 096010 (2010); L. Del Debbio, Proc. Sci. LATTICE2010, 004 (2010); R. Horsley et al., Phys. Lett. B 728, 1 (2014)

[8] S. Weinberg, Physica A 96, 327 (1979)

[9] S.L. Adler, J.C. Collins and A. Duncan, Phys. Rev. D 15, 1712 (1977); P. Minkowski, Berne PRINT-76-0813, September 1976; N.K. Nielsen, Nucl. Phys. B 120, 212 (1977); J.C. Collins, A. Duncan and S.D. Joglekar, Phys. Rev. D 16, 438 (1977)

[10] A. Calle Cordón and E. Ruiz Arriola, AIP Conf. Proc. 1030, 334 (2008); Phys. Rev. C 81, 044002 (2010)

[11] R.J. Crewther, Phys. Rev. Lett. 28, 1421 (1972)

[12] M.S. Chanowitz and J. Ellis, Phys. Lett. B 40, 397 (1972); Phys. Rev. D 7, 2490 (1973)

[13] R.J. Crewther, Nucl. Phys. B 264, 277 (1986)

[14] M. Hoferichter, D.R. Phillips and C. Schat, Eur. Phys. J. C 71, 1743 (2011) 